3

5

6

7

8

9

10

11

12

13

14

15

16

17

18

19

20

21

22

23

24

25

26

27

28

29

30

31

32

33

34

\title{
Prohibitin and mitochondrial biology
}

\author{
Marta Artal-Sanz ${ }^{1}$ and Nektarios Tavernarakis ${ }^{2 *}$
}

${ }^{1}$ Instituto de Biomedicina de Valencia, CSIC, 46010 Valencia, Spain

${ }^{2}$ Institute of Molecular Biology and Biotechnology, Foundation for Research and Technology,

Heraklion 71110, Crete, Greece

8 *Corresponding author: Tavernarakis, N. (tavernarakis@imbb.forth.gr).

Institute of Molecular Biology and Biotechnology

Foundation for Research and Technology - Hellas

N. Plastira 100, Vassilika Vouton, PO Box 1385

Heraklion 70013, Crete, GREECE

tel: +302810 391066 | fax: +302810391067 


\section{Abstract}

Prohibitins are ubiquitous, evolutionarily conserved proteins that are mainly localized in mitochondria. The mitochondrial prohibitin complex comprises two subunits, PHB1 and

PHB2. These two proteins assemble into a ring-like macromolecular structure at the inner mitochondrial membrane and are implicated in diverse cellular processes, from mitochondrial biogenesis and function to cell death and replicative senescence. In humans, prohibitins have been associated with various types of cancer. While their biochemical function remains poorly understood, studies in organisms ranging from yeast to mammals have provided significant insight on the role of the prohibitin complex in mitochondrial biogenesis and metabolism. Here we review the recent studies and discuss their implications towards deciphering the function of prohibitins in mitochondria.

\section{The mitochondrial prohibitin complex}

The eukaryotic mitochondrial PHB complex comprises two highly homologous subunits, PHB1 and PHB2 (around 50\% amino acid sequence identity and 60\% similarity). The first mammalian prohibitin (PHB1) was identified as a potential tumour suppressor with anti-proliferative activity [1], hence named prohibitin. This activity was later attributed to the 3'-UTR of the prohibitin mRNA and found to be unrelated to the function of the protein itself [2]. The second prohibitin (PHB2) was isolated bound to the IgM antigen receptor, together with PHB1. Thus, both proteins were also named $\underline{\mathrm{B}}$-cell-receptor complex-associated proteins (BAP32 and BAP37) [3]. In addition, PHB2 was identified as a repressor of nuclear estrogen receptor activity (termed REA) [4]. Extensive and rapidly accumulating evidence suggests that both prohibitin proteins function mainly within mitochondria [5-8]; reviewed in $[9,10]$. Nevertheless, a number of diverse cellular functions have also been attributed to both PHB1 and PHB2 in other cellular compartments. These include a role in cell cycle progression, regulation of transcription and cell surface signalling (Box 1; reviewed in [11-13]).

62 form a macromolecular structure of approximately $1 \mathrm{MDa}$ at the mitochondrial inner membrane. 
This high molecular weight complex has been identified in yeast, $C$. elegans and mammals [1416]. Prohibitin homodimers have not been detected $[17,18]$. Instead, PHB1 and PHB2 associate with each other to form a heterodimeric building block [17]. About 12 to $16 \mathrm{PHB}$ heterodimers associate to form a ring-like structure at the mitochondrial inner membrane [17] with a diameter of $20-25 \mathrm{~nm}$ [18]. The PHB complex is anchored in the mitochondrial inner membrane through $\mathrm{N}$-terminal hydrophobic regions, present in both PHB1 and PHB2. For yeast PHB2, the transmembrane domain prediction algorithm TMHMM

(http://www.cbs.dtu.dk/services/TMHMM/) predicts a transmembrane helix at positions 37-59, which leaves 36 amino acids at the matrix side with most of the protein facing the intermembrane space. The homologous helical site at the $\mathrm{N}$ terminus of PHB1 is shorter and may not fulfil the requirements for transmembrane spanning helices. For this reason, PHB1 is considered to be membrane associated (Figure 1) [17]. Complex formation depends on both PHB subunits. Depletion of either PHB1 or PHB2 results in the absence of the complex, while the counterpart mRNA is still present. This indicates interdependence at the level of protein complex formation $[5,14,19,20]$. However, detailed structural data about this highly conserved protein complex is still lacking. (Figure 1). The PHB complex has been implicated in regulating membrane protein degradation by the mitochondrial m-AAA protease [16], it has been proposed to function as a holdase/unfoldase chaperone, which holds and stabilizes unassembled membrane proteins [10, 15] and potentially also plays a role in stabilizing the mitochondrial genome [21-23]. In addition, the PHB complex has been implicated in mitochondrial morphogenesis [5], functioning as a scaffold that recruits membrane proteins to a specific lipid environment [24]. Here we review the mitochondrial functions attributed to the PHB complex and focus on their implications for ageing and disease.

\section{Life without prohibitin}

94 Disruption of the PHB complex in Saccharomyces cerevisiae decreases replicative lifespan of yeast cells but does not result in any other observable growth phenotype under laboratory 

conditions $[19,25]$. The shortening of yeast replicative lifespan is accompanied by defects in the mitochondrial membrane potential, extended cell division time and other characteristic morphological changes of ageing cells [25]. Prohibitin depletion does not alter the chronological lifespan of non-dividing ( $\mathrm{G}_{0}$-arrested) cells, although phb-null mutants in stationary phase tend to lose respiratory capacity, which has been associated with deletions of the mitochondrial genome (the [rho'] phenotype) [26]. Increased frequency of [rho']-cell generation can only be detected in old, non-dividing and not in young phb-null mother cells [19, 27]. Similarly, only old phb-null mother cells at the end of their replicative lifespan show defective mitochondrial segregation and aberrant mitochondrial morphology [26]. By contrast, no mitochondrial morphology defects have been detected in younger phb-null cells $[19,27]$. This suggests that phb-null yeast cells undergo premature ageing, probably due to a slight, but cumulative decline in cellular metabolic capacity.

In contrast to the relatively marginal observable effects in yeast, severe phenotypes are associated with prohibitin deficiency in multicellular organisms (see also Box 2). In Caenorhabditis elegans and in mice, prohibitins are required for embryonic development $[5,14$, 20, 28]. Similarly, prohibitins are required for plant development [29, 30]. Post-embryonic depletion of prohibitins in C. elegans results in pronounced germline defects such as diminished oocyte production with smaller brood size [14]. Both embryonic and postembryonic effects observed in C. elegans indicate that PHB proteins are specifically required in tissues that undergo cellular proliferation. Extensive distortion of mitochondrial morphology is observed following reduction of prohibitin expression in C. elegans body wall muscles [14]. During postembryonic development, nematode muscle cells do not proliferate but rather grow in size. Normally, the number of mitochondria per cell increases to meet energy requirements of muscle growth. The effects of prohibitin depletion suggest that the prohibitin complex plays an important role in maintaining mitochondrial membrane integrity in these cells. Deletion of PHB2 in mouse embryonic fibroblasts (MEFs) results in severely impaired cellular proliferation [5]. Accordingly, it appears likely that tissues that rely heavily on mitochondrial function, i.e. proliferating cells, are more susceptible to lack of prohibitin. 
128 diauxic shift, when yeast cells switch from non-oxidative to oxidative metabolism. Furthermore,

129 yeast mutants defective in the synthesis of the mitochondrially encoded Cox $1 p$ subunit show

130 increased levels of the PHB complex [10]. Finally, inhibition of mitochondrial translation results

131 in increased PHB expression in human cells and in C. elegans [14, 31]. Mitochondrial and

132 nuclear encoded subunits of the respiratory chain need to assemble stoichiometrically in the

133 mitochondrial membrane, and imbalances between subunits represent a threat for membrane

134 integrity and mitochondrial function. For example, production of reactive oxygen species (ROS)

135 might increase, as well as leakage of $\mathrm{H}^{+}$and ROS. Therefore, these findings support a role for

136 the PHB complex as a holdase type of chaperone specifically required in situations of

137 mitochondrial stress [10].

138

Extensive studies on the expression patterns of both PHB proteins in mammalian

140 tissues and during murine development also support a role for prohibitins in regulating

141 mitochondrial metabolism. PHB proteins are highly expressed in cells that rely heavily on

142 mitochondrial function, including neurons, muscle, heart, liver, renal tubules, adrenal cortex,

143 brown adipocytes and pancreatic islet cells [31]. These tissues are often particularly susceptible

144 to mitochondrial dysfunction [32]. Plant prohibitins are predominantly expressed in proliferating

145 tissues [30]. Similarly, PHB proteins are expressed at higher levels in mammalian proliferating

146 cells, including neoplastic tissues (see also Box 2) [31, 33].

\section{Prohibitin and mitochondrial dynamics}

150 Mitochondria are highly dynamic structures that continuously fuse and divide to adjust the

151 shape and distribution of the mitochondrial network depending on cell type and energy

152 demands. Mitochondrial dynamics play a critical role in cell physiology. Conserved protein

153 machineries located in the outer and inner membrane of mitochondria regulate fusion and

154 fission events and include several dynamin-like GTPases [34]. Among them, mitofusins (Mfn1,

155 Mfn2) and optic atrophy 1 protein (OPA1) are required for mitochondrial fusion, and dynamin-

156 related protein (DRP1) is required for mitochondrial fission. 
Loss of prohibitins was first shown to severely affect mitochondrial morphology in $C$.

elegans body wall muscle cells [14]. In normal muscle cells, mitochondria appear tubular,

160 elongated, and well structured, running parallel to the body axis and often parallel to the myofibrils. Upon prohibitin depletion, mitochondria appear fragmented and disorganized [14].

162 Similarly, loss of prohibitins results in the accumulation of fragmented mitochondria in MEFs and HeLa cells $[5,35]$. A possible mechanism for prohibitin-depletion mediated mitochondrial

164 fragmentation has been put forward after the discovery that PHB2 deletion results in OPA1 destabilization $[5,35]$. OPA1 resides in the mitochondrial inner membrane and has a key role in mitochondrial fusion and cristae morphology. Electron microscopy analysis of PHB2-depleted MEFs revealed severe defects in lamellar cristae formation [5]. Similarly, lack of cristae has been reported in plant mitochondrial depleted of prohibitin [36]. The mitochondrial fragmentation and highly disorganised cristae of PHB2 depleted MEFs strikingly resembles the mitochondrial morphology observed after OPA1 down-regulation [5, 37]. processing involving $\mathrm{m}$ - and i-AAA mitochondrial proteases [39-41]. Five isoforms have been described in MEFs and HeLa cells (two long isoforms, L-OPA1, and three short isoforms, SOPA1). Deletion of PHB2 in MEFs results in the specific loss of L-OPA1 isoforms and an altered pattern in the accumulation of S-OPA1 isoforms [5]. In fact, defects in MEFs lacking PHB2 (mitochondrial fragmentation, aberrant cristae morphology, impaired cellular proliferation and increased cytochrome $c$ release after apoptotic stimulation), were all partially rescued by overexpressing a non-cleavable L-OPA1 isoform [5], while expression of an S-OPA1 isoform

180 had no effect. These observations suggest that the central role of prohibitin in mitochondria is to regulate OPA1 processing. If so, expression of L-OPA1 is expected to bypass PHB-depletion.

182 Because the activity of OPA1 depends on both L- and S-OPA1 isoforms, it would be interesting to see if suppression of PHB deficiency is enhanced by the simultaneous expression of both non-cleavable L-OPA1 and S-OPA1 isoforms. Further investigation of the mechanism by which the PHB complex affects mitochondrial fusion and the proposed stabilisation of OPA1 will shed light on these questions dynamics. A mitochondrial stomatin-like protein (SLP-2/Stoml2) has been shown to interact with 
190 prohibitins in the mitochondrial inner membrane [42]. Stomatins contain an erythrocyte band-7

191 motif and belong to the SPFH family of proteins, which includes stomatins, prohibitins, flotillins

192 and $\underline{H}$ fIK/C bacterial proteases [43]. Depleting HeLa cells of SLP-2 results in increased

193 proteolysis of PHB1, PHB2, and subunits of respiratory chain complexes I and IV. The stability

194 of prohibitins upon mitochondrial stress partially depends on SLP-2 [42], and PHB expression is

195 increased following mitochondrial stress [14, 15, 31, 44]. Recently, SLP-2/Stoml2 was shown to

196 interact with Mfn2 [45]. Apparently, only a small portion of Mfn2 is involved in forming Mfn2-

197 Stoml2 hetero-oligomers, and reduction of Stoml2 does not affect mitochondrial morphology in

198 HeLa cells [45]. Nevertheless, depletion of the C. elegans orthologue of SLP-2/Stoml2 (STL-1)

199 results in mitochondrial fragmentation [46]. Mfn1 and Mfn2 interact with Opa1 to mediate

200 mitochondrial fusion [47]. Because the major domains of Mfns are orientated towards the

201 cytoplasm, it is likely that the interaction between Mfns and OPA1 at the mitochondrial inner

202 membrane is not direct, but mediated by additional proteins, such as SLP-2/Stoml2 and/or

203 prohibitins.

A partial structure for the PHB complex was derived in an attempt to elucidate its molecular mechanism of action [17]. The best-fit 3D structure was the four-helical bundle structure of the t-SNAREs syntaxin $1 \mathrm{~A}$ and the yeast Sso1p [17]. While one should be careful when modelling unknown structures with less than $30 \%$ homology to known structures, it is tempting to consider that proteins with a similar fold might share some functional properties.

210 SNARE proteins are key components of protein complexes that drive membrane fusion [48].

211 Mfns and OPA1 do not resemble SNARE proteins, which suggests, mitochondrial membrane

212 fusion occurs by a distinct mechanism. However, recent discoveries open the possibility that the

213 basic mechanism of mitochondrial fusion might be more similar to the fusion mechanisms used

214 by other organelles than previously believed [49]. Classical Phospholipase D (PLD) cleaves

215 phosphatidylcholine to produce phosphatidic acid; a fusogenic lipid important for SNARE

216 mediated membrane fusion [50]. A mitochondrial phospholipase D (MitoPLD) required for

217 mitochondrial fusion has recently been identified [49]. MitoPLD promotes transmitochondrial

218 membrane adherence in an Mfn-dependent manner by hydrolysing cardiolipin to generate

219 phosphatidic acid. MitoPLD IS located at the mitochondrial outer membrane with its catalytic

220 domain exposed to the cytosol. Because cardiolipin is synthesized in the inner membrane, it needs to be transported to the outer membrane, probably through contact sites between the two 
membranes. In this context, it is worth noting that mutations impairing the biosynthetic machinery of PtdEtn and cardiolipin show synthetic lethality when combined with prohibitin depletion in S. cerevisiae $[19,24]$ and a role for the PHB complex in lipid partitioning has been suggested [24]. Taken together, the proposed similarity of the PHB complex with SNARE proteins [17], its interaction with SLP-2/Stoml2 [42] and the interaction of SLP-2/Stoml2 with Mfn [45], the Mfn-dependent role of MitoPLD in mitochondrial fusion [49] and the possible role of prohibitins as lipid membrane organizers [24] suggest that the PHB complex might play a more direct role in mitochondrial membrane fusion that suspected (Figure 1). Members of the SPFH family have been found in lipid rafts [52] or directly interacting with lipids [53]. They contain a conserved domain next to the predicted $\mathrm{N}$-terminal transmembrane stretch

234 that has been called the PHB domain [52]. Although the function of this domain is not clear, it has been proposed to bind lipids or lipid motifs [54]. In this context, the PHB complex could have a role in keeping the mitochondrial outer and inner membrane in close proximity, or even both mitochondrial inner membranes if located at mitochondrial cristae (Figure 1)

\section{Prohibitin and oxidative phosphorylation}

241 Studies in S. cerevisiae suggest a role for prohibitin in the assembly of the oxidative

242 phosphorylation (OXPHOS) system. As mentioned previusly, the PHB complex might function 243 as a holdase/unfoldase type of chaperone and in membrane quality control in association with

244 the mitochondrial $m$-AAA proteases $[15,16]$. Although there is no clear evidence for either an association of the PHB complex with assembly intermediates or for an essential role in the biogenesis of the OXPHOS system, experimental findings support a role for the PHB complex in handling mitochondrial membrane proteins and in the stability of the OXPHOS system. in phb-null yeast cells [16, 27], and overexpression of the PHB complex in yeast results in stabilization of newly synthesized mitochondrial encoded membrane subunits [15]. Prohibitins 
complex I in mammals $[55,56]$. Expression of prohibitins increases in situations of imbalance between nuclear- and mitochondrial-encoded OXPHOS proteins in yeast, C. elegans and mammals [14, 15, 31, 42, 44]. In addition, depletion of PHB-2 in C. elegans signals the mitochondrial unfolded protein response and strongly activates mitochondrial chaperones [57, 58]. Moreover, reduced cytosolic protein synthesis, which results in reduced load of cytosolic proteins onto the mitochondrial inner membrane, suppresses mitochondrial degeneration of phb-null yeast cells [8].

Yeast cells depleted of PHB genes have reduced mitochondrial membrane potential $[24,25]$. Similarly, low prohibitin levels in plants result in reduced membrane potential and oxygen consumption [36]. Furthermore, knockdown of both phb-1 and phb-2 genes in C. elegans results in slightly reduced oxygen consumption [14]. Endothelial, PHB1-depleted cells have depolarized mitochondria and show reduced complex I activity. The activities of complexes II and III were normal, while complex IV was not measured in these experiments. Oxygen consumption was maintained apparently by a compensatory mechanism that allowed electron flow through complexes II and III [7]. However, in MEFs depleted of PHB2, membrane potential, ATP levels, oxygen consumption and electron transport chain activities were normal [5], suggesting cell-type specific differences in the requirement of the PHB complex for appropriate OXPHOS function.

Prohibitins have also been functionally and physically associated to mitochondrial DNA (mtDNA). Yeast cells lacking mtDNA [rho $\left./ \mathrm{rho}^{0}\right]$ become petite-negative after Phb1p depletion [59]. This phenotype is genetic background-dependent since strains deleted for PHB1 in a different background are viable after mtDNA loss [19]. Physical association of prohibitins to mtDNA nucleoids has been reported in Xenopus oocytes and in HeLa cells [21-23]. RNAimediated down-regulation of PHB1 in HeLa cells results in altered organization and reduced copy number of mtDNA, attributed to the destabilisation of the mitochondrial transcription factor A (TFAM) [22], which is essential for mtDNA maintenance [60]. How do prohibitins affect mtDNA when most of the prohibitin complex faces the intermembrane space? It has been suggested that the PHB complex might interact with mtDNA via protein components of mitochondrial nucleoids. Alternative explanations also exist. Mitochondrial nucleoids are attached to the mitochondrial inner membrane. It is therefore, possible that alterations of protein and/or lipid 
composition in the mitochondrial inner membrane after PHB depletion, as well as the pronounced defect in cristae morphology observed, may affect the attachment of nucleoids to the inner membrane. In agreement with this, loss of mtDNA is also observed in patients with OPA1 mutations [61] and in yeast cells depleted of the OPA1 homologue Mgm1 [62]. Because mtDNA encodes for essential subunits of the OXPHOS system, regardless whether prohibitins depletion affects directly or indirectly mtDNA, the net result will likely be defects in OXPHOS. increase the production of free radicals by disrupting OXPHOS. Indeed, lack of PHB1 in endothelial cells results in increased levels of reactive oxygen species (ROS), which has been associated with a senescent-like phenotype [7]. Prohibitin depletion in C. elegans and in plants causes increased sensitivity to oxidative stress [14, 36], indicating elevated endogenous ROS

297 formation. Similarly, nematodes deleted for eat-3, the orthologue of OPA1, are sensitive to free radical-induced damage. Expression of the mitochondrial matrix Fe/Mn-superoxide dismutase, SOD-2, is increased in eat-3 mutants, and disruption of the sod-2 gene severely compromises survival of eat-3 mutant nematodes. Interestingly, increased ROS production and mitochondrial fragmentation has also been reported in Drosophila OPA1 mutants [63].

\section{Conclusions and challenges}

305 Despite decades of investigation, the function of the PHB complex still remains a mystery. Is the

306 PHB complex a holdase/unfoldase chaperone that protects the membrane from unfolded and 307 unassembled proteins, assisting their degradation? Or, do prohibitins act as protein and/or DNA

308 scaffolds? Does the PHB complex have a direct role in mitochondrial membrane

309 morphogenesis? Much still remains to be understood about this highly evolutionarily conserved

310 inner mitochondrial membrane complex. New ideas have been put forward, including a putative

311 role in mitochondrial genome stability, mitochondrial membrane morphology or in mitochondrial 312 membrane fusion (Figure 2).

314 The recent observation that lack of the PHB complex results in a dramatic

315 destabilisation of OPA1 [5, 35] provides new insight into the effect of PHB depletion on 
316 mitochondrial ultrastructure and cristae morphology. Altered cristae morphology caused by lack

317 of prohibitin [5, 36], may underlie the destabilisation of mitochondrial transcription factor $A$

318 (TFAM) and mtDNA, which will ultimately result in defective OXPHOS and other mitochondrial

319 metabolic pathways, However, additional evidence suggests that the PHB complex has

320 functions beyond OPA1 stabilisation. Deletion of the C. elegans orthologue of OPA1 (EAT-3)

321 results in viable animals, whereas prohibitin deficiency is lethal [14]; see also

322 http://www.wormbase.org/). This dramatic difference suggests that prohibitins have additional

323 functions, independent of OPA1 stabilisation and consequent maintenance of cristae

324 morphology.

Immuno-electron microscopy on mammalian cells shows that the OPA1 protein is mostly distributed throughout cristae, with only a small portion localized to the boundary space between mitochondrial inner and outer membranes [37]. In this context, it would be interesting to determine the specific mitochondrial sub-localisation of the PHB complex. Localisation at the inner boundary membrane would indicate a role in cristae formation or in mediating connections between the inner and the outer mitochondrial membranes, and would also be in agreement with its proposed role as holdase in the process of OXPHOS complex assembly (Figure 1). Assembly of the respiratory chain and ATP synthase requires both proteins imported from the cytosol and mitochondrially synthesised subunits. While cristal membranes seem to be the principal site of oxidative phosphorylation [64], OXPHOS complexes are more likely to assemble in the inner boundary membrane where mitochondrial- and nuclear-encoded subunits first encounter each other [65]. Additionally, localisation at cristae would suggest a role in cristae morphology or even in maintaining cristae membranes in close proximity. These are testable alternative hypotheses in the quest of further investigating the role of the PHB complex.

342 mitochondrial membranes and is essential for cellular proliferation. Nevertheless, how lack of 343 prohibitin affects mitochondrial morphology and how mitochondrial morphology defects impair 344 cellular proliferation remain unknown. Mutations in OXPHOS components have been reported 345 to inhibit cell division through AMP kinase and cyclin E [66]. Additionally, mitochondria associate 346 with spindle poles and have a role in spindle positioning and alignment in eukaryotic cells and in 347 C. elegans $[67,68]$. Knock down of PHB2 in HeLa cells affects sister chromatid cohesion and 
348 spindle formation during mitosis [69]. It would be interesting to determine whether impaired

349 proliferation of cells lacking prohibitins is due to defects on mitochondrial energy metabolism,

350 due to defective mitochondrial morphology, or both.

351

Despite the considerable recent progress towards deciphering the function of

353 prohibitins, the above seemingly disparate observations underline our poor overall

354 understanding of the PHB complex. Resolving the three-dimensional structure of the PHB

355 complex will certainly help in defining its function at the molecular level. In addition, the genetic

356 dissection of prohibitin in animal models holds promise for unravelling novel mechanisms by

357 which mitochondrial biogenesis and function influence fundamental cellular processes including

358 pathogenesis and ageing.

359

360

\section{Acknowledgements}

362 We thank Julio Mateos Langerak for help with the figures. We gratefully acknowledge the

363 contributions of those investigators whose work we did not include in this review, owing to space

364 limitations. This work was supported by the European Commission Coordination Action ENINET

365 (contract number LSHM-CT-2005-19063).

366 


\section{References}

1. McClung, J.K., et al., Isolation of a cDNA that hybrid selects antiproliferative mRNA from rat liver. Biochem Biophys Res Commun, 1989. 164(3): p. 1316-22.

2. Jupe, E.R., et al., Prohibitin in breast cancer cell lines: loss of antiproliferative activity is linked to 3' untranslated region mutations. Cell Growth Differ, 1996. 7(7): p. 871-8.

3. Terashima, M., et al., The IgM antigen receptor of $B$ lymphocytes is associated with prohibitin and a prohibitin-related protein. EMBO J, 1994. 13(16): p. 3782-92.

4. Montano, M.M., et al., An estrogen receptor-selective coregulator that potentiates the effectiveness of antiestrogens and represses the activity of estrogens. Proc Natl Acad Sci U S A, 1999. 96(12): p. 6947-52.

5. Merkwirth, C., et al., Prohibitins control cell proliferation and apoptosis by regulating OPA1dependent cristae morphogenesis in mitochondria. Genes Dev, 2008. 22(4): p. 476-88.

6. Ross, J.A., Z.S. Nagy, and R.A. Kirken, The PHB1/2 phosphocomplex is required for mitochondrial homeostasis and survival of human T cells. J Biol Chem, 2008. 283(8): $p$. 4699-713.

7. Schleicher, M., et al., Prohibitin-1 maintains the angiogenic capacity of endothelial cells by regulating mitochondrial function and senescence. J Cell Biol, 2008. 180(1): p. 101-12.

8. Wang, X., et al., Reduced cytosolic protein synthesis suppresses mitochondrial degeneration. Nat Cell Biol, 2008. 10(9): p. 1090-7.

9. Merkwirth, C. and T. Langer, Prohibitin function within mitochondria: Essential roles for cell proliferation and cristae morphogenesis. Biochim Biophys Acta, 2009. 1793(1): p. 27-32.

10. Nijtmans, L.G., et al., The mitochondrial PHB complex: roles in mitochondrial respiratory complex assembly, ageing and degenerative disease. Cell Mol Life Sci, 2002. 59(1): p. 14355.

11. Mishra, S., L.C. Murphy, and L.J. Murphy, The Prohibitins: emerging roles in diverse functions. J Cell Mol Med, 2006. 10(2): p. 353-63.

12. Mishra, S., et al., Prohibitin: a potential target for new therapeutics. Trends Mol Med, 2005. 11(4): p. 192-7.

13. Rajalingam, K. and T. Rudel, Ras-Raf signaling needs prohibitin. Cell Cycle, 2005. 4(11): p. 1503-5.

14. Artal-Sanz, M., et al., The mitochondrial prohibitin complex is essential for embryonic viability and germline function in Caenorhabditis elegans. J Biol Chem, 2003. 278(34): p. 32091-9.

15. Nijtmans, L.G., et al., Prohibitins act as a membrane-bound chaperone for the stabilization of mitochondrial proteins. EMBO J, 2000. 19(11): p. 2444-51.

16. Steglich, G., W. Neupert, and T. Langer, Prohibitins regulate membrane protein degradation by the m-AAA protease in mitochondria. Mol Cell Biol, 1999. 19(5): p. 3435-42.

17. Back, J.W., et al., A structure for the yeast prohibitin complex: Structure prediction and evidence from chemical crosslinking and mass spectrometry. Protein Sci, 2002. 11(10): p. 2471-8.

18. Tatsuta, T., K. Model, and T. Langer, Formation of membrane-bound ring complexes by prohibitins in mitochondria. Mol Biol Cell, 2005. 16(1): p. 248-59.

19. Berger, K.H. and M.P. Yaffe, Prohibitin family members interact genetically with mitochondrial inheritance components in Saccharomyces cerevisiae. Mol Cell Biol, 1998. 18(7): p. 4043-52.

20. He, B., et al., A repressive role for prohibitin in estrogen signaling. Mol Endocrinol, 2008. 22(2): p. 344-60.

21. Bogenhagen, D.F., D. Rousseau, and S. Burke, The layered structure of human mitochondrial DNA nucleoids. J Biol Chem, 2008. 283(6): p. 3665-75.

22. Kasashima, K., et al., Human prohibitin 1 maintains the organization and stability of the mitochondrial nucleoids. Exp Cell Res, 2008. 314(5): p. 988-96.

23. Wang, Y. and D.F. Bogenhagen, Human mitochondrial DNA nucleoids are linked to protein folding machinery and metabolic enzymes at the mitochondrial inner membrane. J Biol Chem, 2006. 281(35): p. 25791-802.

24. Osman, C., et al., The genetic interactome of prohibitins: coordinated control of cardiolipin and phosphatidylethanolamine by conserved regulators in mitochondria. J Cell Biol, 2009. 184(4):583-96.

25. Coates, P.J., et al., The prohibitin family of mitochondrial proteins regulate replicative lifespan. Curr Biol, 1997. 7(8): p. 607-10. 
26. Piper, P.W., et al., The shortened replicative life span of prohibitin mutants of yeast appears to be due to defective mitochondrial segregation in old mother cells. Aging Cell, 2002. 1(2): p. 149-57.

27. Birner, R., et al., Synthetic lethal interaction of the mitochondrial phosphatidylethanolamine biosynthetic machinery with the prohibitin complex of Saccharomyces cerevisiae. Mol Biol Cell, 2003. 14(2): p. 370-83.

28. Park, S.E., et al., Genetic deletion of the repressor of estrogen receptor activity (REA) enhances the response to estrogen in target tissues in vivo. Mol Cell Biol, 2005. 25(5): p. 1989-99.

29. Chen, J.C., C.Z. Jiang, and M.S. Reid, Silencing a prohibitin alters plant development and senescence. Plant J, 2005. 44(1): p. 16-24.

30. Van Aken, O., et al., Mitochondrial type-I prohibitins of Arabidopsis thaliana are required for supporting proficient meristem development. Plant J, 2007. 52(5): p. 850-64.

31. Coates, P.J., et al., Mammalian prohibitin proteins respond to mitochondrial stress and decrease during cellular senescence. Exp Cell Res, 2001. 265(2): p. 262-73.

32. Wallace, D.C., Mitochondrial diseases in man and mouse. Science, 1999. 283(5407): p. $1482-8$.

33. Czarnecka, A.M., et al., Mitochondrial chaperones in cancer: from molecular biology to clinical diagnostics. Cancer Biol Ther, 2006. 5(7): p. 714-20.

34. Detmer, S.A. and D.C. Chan, Functions and dysfunctions of mitochondrial dynamics. Nat Rev Mol Cell Biol, 2007. 8(11): p. 870-9.

35. Kasashima, K., et al., Mitochondrial functions and estrogen receptor-dependent nuclear translocation of pleiotropic human prohibitin 2. J Biol Chem, 2006. 281(47): p. 36401-10.

36. Ahn, C.S., et al., Prohibitin is involved in mitochondrial biogenesis in plants. Plant J, 2006. 46(4): p. 658-67.

37. Griparic, L., et al., Loss of the intermembrane space protein Mgm1/OPA1 induces swelling and localized constrictions along the lengths of mitochondria. J Biol Chem, 2004. 279(18): p. $18792-8$.

38. Delettre, C., et al., Mutation spectrum and splicing variants in the OPA1 gene. Hum Genet, 2001. 109(6): p. 584-91.

39. Cipolat, S., et al., Mitochondrial rhomboid PARL regulates cytochrome $c$ release during apoptosis via OPA1-dependent cristae remodeling. Cell, 2006. 126(1): p. 163-75.

40. Duvezin-Caubet, S., et al., OPA1 processing reconstituted in yeast depends on the subunit composition of the m-AAA protease in mitochondria. Mol Biol Cell, 2007. 18(9): p. 3582-90.

41. Griparic, L., T. Kanazawa, and A.M. van der Bliek, Regulation of the mitochondrial dynaminlike protein Opa1 by proteolytic cleavage. J Cell Biol, 2007. 178(5): p. 757-64.

42. Da Cruz, S., et al., SLP-2 interacts with prohibitins in the mitochondrial inner membrane and contributes to their stability. Biochim Biophys Acta, 2008. 1783(5): p. 904-11.

43. Tavernarakis, N., M. Driscoll, and N.C. Kyrpides, The SPFH domain: implicated in regulating targeted protein turnover in stomatins and other membrane-associated proteins. Trends Biochem Sci, 1999. 24(11): p. 425-7.

44. Nijtmans, L.G., et al., Shy1p occurs in a high molecular weight complex and is required for efficient assembly of cytochrome c oxidase in yeast. FEBS Lett, 2001. 498(1): p. 46-51.

45. Hajek, P., A. Chomyn, and G. Attardi, Identification of a novel mitochondrial complex containing mitofusin 2 and stomatin-like protein 2. J Biol Chem, 2007. 282(8): p. 5670-81.

46. Ichishita, R., et al., An RNAi screen for mitochondrial proteins required to maintain the morphology of the organelle in Caenorhabditis elegans. J Biochem, 2008. 143(4): p. 44954.

47. Guillery, O., et al., Metalloprotease-mediated OPA1 processing is modulated by the mitochondrial membrane potential. Biol Cell, 2008. 100(5): p. 315-25.

48. Jahn, R. and R.H. Scheller, SNAREs--engines for membrane fusion. Nat Rev Mol Cell Biol, 2006. 7(9): p. 631-43.

49. Choi, S.Y., et al., A common lipid links Mfn-mediated mitochondrial fusion and SNAREregulated exocytosis. Nat Cell Biol, 2006. 8(11): p. 1255-62.

50. Davletov, B., E. Connell, and F. Darios, Regulation of SNARE fusion machinery by fatty acids. Cell Mol Life Sci, 2007. 64(13): p. 1597-608.

51. Browman, D.T., M.B. Hoegg, and S.M. Robbins, The SPFH domain-containing proteins: more than lipid raft markers. Trends Cell Biol, 2007. 17(8): p. 394-402.

52. Rivera-Milla, E., C.A. Stuermer, and E. Malaga-Trillo, Ancient origin of reggie (flotillin), reggie-like, and other lipid-raft proteins: convergent evolution of the SPFH domain. Cell Mol Life Sci, 2006. 63(3): p. 343-57.

53. Huber, T.B., et al., Podocin and MEC-2 bind cholesterol to regulate the activity of associated ion channels. Proc Natl Acad Sci U S A, 2006. 103(46): p. 17079-86. 
488

489

490

491

492

493

494

495

496

497

498

499

500

501

502

503

504

505

506

507

508

509

510

511

512

513

514

515

516

517

518

519

520

521

522

523

524

525

526

527

528

529

530

531

532

533

534

535

536

537

538

539

540

541

542

543

544

545

546

547

548

549

550
54. Morrow, I.C. and R.G. Parton, Flotillins and the PHB domain protein family: rafts, worms and anaesthetics. Traffic, 2005. 6(9): p. 725-40.

55. Bourges, I., et al., Structural organization of mitochondrial human complex I: role of the ND4 and ND5 mitochondria-encoded subunits and interaction with prohibitin. Biochem J, 2004. 383(Pt. 3): p. 491-9.

56. Taylor, S.W., et al., Characterization of the human heart mitochondrial proteome. Nat Biotechnol, 2003. 21(3): p. 281-6.

57. Benedetti, C., et al., Ubiquitin-like protein 5 positively regulates chaperone gene expression in the mitochondrial unfolded protein response. Genetics, 2006. 174(1): p. 229-39.

58. Yoneda, T., et al., Compartment-specific perturbation of protein handling activates genes encoding mitochondrial chaperones. J Cell Sci, 2004. 117(Pt 18): p. 4055-66.

59. Dunn, C.D., et al., A genomewide screen for petite-negative yeast strains yields a new subunit of the i-AAA protease complex. Mol Biol Cell, 2006. 17(1): p. 213-26.

60. Chen, X.J. and R.A. Butow, The organization and inheritance of the mitochondrial genome. Nat Rev Genet, 2005. 6(11): p. 815-25.

61. Kim, J.Y., et al., Mitochondrial DNA content is decreased in autosomal dominant optic atrophy. Neurology, 2005. 64(6): p. 966-72.

62. Jones, B.A. and W.L. Fangman, Mitochondrial DNA maintenance in yeast requires a protein containing a region related to the GTP-binding domain of dynamin. Genes Dev, 1992. 6(3): p. 380-9.

63. Yarosh, W., et al., The molecular mechanisms of OPA1-mediated optic atrophy in Drosophila model and prospects for antioxidant treatment. PLoS Genet, 2008. 4(1): p. e6.

64. Gilkerson, R.W., J.M. Selker, and R.A. Capaldi, The cristal membrane of mitochondria is the principal site of oxidative phosphorylation. FEBS Lett, 2003. 546(2-3): p. 355-8.

65. Reichert, A.S. and W. Neupert, Contact sites between the outer and inner membrane of mitochondria-role in protein transport. Biochim Biophys Acta, 2002. 1592(1): p. 41-9.

66. Mandal, S., et al., Mitochondrial regulation of cell cycle progression during development as revealed by the tenured mutation in Drosophila. Dev Cell, 2005. 9(6): p. 843-54.

67. Dinkelmann, M.V., et al., SPD-3 is required for spindle alignment in Caenorhabditis elegans embryos and localizes to mitochondria. Genetics, 2007. 177(3): p. 1609-20.

68. Kruger, N. and I.M. Tolic-Norrelykke, Association of mitochondria with spindle poles facilitates spindle alignment. Curr Biol, 2008. 18(15): p. R646-R647.

69. Takata, H., et al., PHB2 protects sister-chromatid cohesion in mitosis. Curr Biol, 2007. 17(15): p. 1356-61.

70. Nuell, M.J., et al., Prohibitin, an evolutionarily conserved intracellular protein that blocks DNA synthesis in normal fibroblasts and HeLa cells. Mol Cell Biol, 1991. 11(3): p. 1372-81.

71. Sharma, A. and A. Qadri, Vi polysaccharide of Salmonella typhi targets the prohibitin family of molecules in intestinal epithelial cells and suppresses early inflammatory responses. Proc Natl Acad Sci U S A, 2004. 101(50): p. 17492-7.

72. Kolonin, M.G., et al., Reversal of obesity by targeted ablation of adipose tissue. Nat Med, 2004. 10(6): p. 625-32.

73. Heron-Milhavet, L., et al., Akt2 is implicated in skeletal muscle differentiation and specifically binds Prohibitin2/REA. J Cell Physiol, 2008. 214(1): p. 158-65.

74. Sun, L., et al., Akt binds prohibitin 2 and relieves its repression of MyoD and muscle differentiation. J Cell Sci, 2004. 117(Pt 14): p. 3021-9.

75. Beckman, K.B. and B.N. Ames, The free radical theory of aging matures. Physiol Rev, 1998. 78(2): p. 547-81.

76. Doonan, R., et al., Against the oxidative damage theory of aging: superoxide dismutases protect against oxidative stress but have little or no effect on life span in Caenorhabditis elegans. Genes Dev, 2008. 22(23): p. 3236-41.

77. Inoue, K., et al., Generation of mice with mitochondrial dysfunction by introducing mouse mtDNA carrying a deletion into zygotes. Nat Genet, 2000. 26(2): p. 176-81.

78. Casari, G., et al., Spastic paraplegia and OXPHOS impairment caused by mutations in paraplegin, a nuclear-encoded mitochondrial metalloprotease. Cell, 1998. 93(6): p. 973-83.

79. Santamaria, E., et al., Functional proteomics of nonalcoholic steatohepatitis: mitochondrial proteins as targets of S-adenosylmethionine. Proc Natl Acad Sci U S A, 2003. 100(6): p. 3065-70.

80. Ferrer, I., et al., Abnormal levels of prohibitin and ATP synthase in the substantia nigra and frontal cortex in Parkinson's disease. Neurosci Lett, 2007. 415(3): p. 205-9.

81. Smalla, K.H., et al., A comparison of the synaptic proteome in human chronic schizophrenia and rat ketamine psychosis suggest that prohibitin is involved in the synaptic pathology of schizophrenia. Mol Psychiatry, 2008. 13(9): p. 878-96. 


\section{Text Boxes}

551

\section{Box 1. Prohibitins outside of mitochondria}

553

554

555

556

557

558

559

560

561

562

563

564

565

566

567

568

569

570

571

572

573

574

575

576

577

578

579

580 activity, hence termed REA [28].

Initially, prohibitin (PHB1) was proposed to play a role in cell cycle progression $[1,70]$.

Later PHB2 was identified as a B-cell receptor associated protein at the plasma membrane [3], and named BAP37 for B-cell associated protein. In addition, both PHB1 and PHB2 were found at the plasma membrane of human intestinal epithelial cells, functioning as a binding site for the Vi capsular polysaccharide of Salmonella typhi [71]. PHB1 has also been found to be the target of a proapoptotic peptide in adipose vasculature [72]. Furthermore, PHB1 has been implicated in mediating cellular Ras-Raf signalling at the membrane [13]. PHB1 has also been shown to modulate transcription in cell-transfection experiments and to bind to a wide range of proteins, including Retinoblastoma (Rb), E2-F, Brg1/Brm and p53 [11, 12]. PHB2 was found to modulate muscle differentiation by binding to AKT $[73,74]$ and also to represses estrogen receptor (ER $\alpha)$

Given the strong interdependence of PHB1 and PHB2 in mitochondria it was puzzling how the proteins alone could be stable in other cellular compartments. Recently, PHB (PHB1) was also shown to repress ER $\alpha$ activity [20]. Interestingly, similarly to mitochondrial prohibitins, PHB and REA interact and stabilise each other in vivo. Reducing the amount of REA results in reduced $\mathrm{PHB}$, and vice versa. Surprisingly, co-expressing both proteins together eliminates the transcriptional effects of the individual proteins. This suggests that heteromers of PHB and REA are inefficient as transcriptional co-repressors. Therefore, PHB and REA might only repress transcription when they are not paired [20]. How these evolutionarily conserved proteins can exert such a variety of functions within the cell is currently not understood. 


\section{Box 2. Prohibitin in ageing and disease}

583 Mammalian cell senescence is accompanied by reduced expression of both PHB proteins. This decrease correlates with a heterogeneous decline in mitochondrial membrane potential during ageing [31]. Studies in yeast provide direct support for the involvement of the PHB complex in the ageing process. Deletion of either or both of the PHB genes shortens the replicative lifespan of yeast by about one third $[19,25]$. Cells deleted for PHB1 and PHB2 show a roughened cell surface and prolonged cell cycle after fewer divisions, compared to wild type, indicating that the normal ageing process has been accelerated in cells lacking the PHB complex [25]. Similarly, depletion of prohibitin shortens the lifespan of petunia flowers [29]. evidence links the PHB complex to mitochondrial function. Thus, it is likely that prohibitin influences longevity by affecting mitochondrial metabolism. Lack of the PHB complex results in increased ROS production [7], and sensitivity to free radicals [14, 36]. Accumulation of cellular damage as a consequence of the production of free radicals has been suggested to drive the ageing process [75] and may be responsible for the reduced lifespan upon PHB depletion. However, recent reports demonstrating lack of correlation between oxidative damage and longevity, challenge the free-radical theory of ageing [76].

600 Myopathies and neuropathies are among the most common types of disorders associated with mitochondrial defects. Other disorders such as diabetes, hearing loss and kidney failure are also caused by mitochondrial dysfunction $[32,77]$. To date, no mutations in the PHB genes have been found to cause human disease. Nevertheless, mutations in the mitochondrial $\mathrm{m}$ AAA-metalloprotease, which interacts with the PHB complex [16] cause hereditary spastic paraplegia in humans [78]. In addition, high levels of prohibitin expression in tumours indicate their potential role in carcinogenesis [33]. Expression of PHB proteins is also elevated in yeast cells devoid of SHY1, the orthologue of SURF1, which is associated with Leigh syndrome [44].

610 Altered expression of PHB1 correlates with loss of mitochondrial function in the liver of knockout

611 mice deficient in S-adenosylmethionine synthesis and in obese patients who are at risk for

612 nonalcoholic steatohepatitis [79]. In addition, abnormal levels of prohibitin have been reported in 
613 Parkinson's disease [80] and in schizophrenia [81]. These findings emphasise the importance

614 of the PHB complex in maintaining mitochondrial homeostasis, which is critical for human

615 health.

616

617

618 Box 3. Glossary

619 IgM: Immunoglobulin M. An antibody that is present on B lymphocytes, which are involved in

620 the humoral immune response. IgM is the primary antibody against $A$ and $B$ antigens on red

621 blood cells.

622

m-AAA protease: Mitochondrial matrix-AAA protease. Member of membrane-bound ATP-

623 dependent proteases that are present in eubacteria, mitochondria and chloroplasts and that can

624 degrade membrane proteins.

625 Replicative lifespan: Yeast cells age chronologically, but also undergo replicative senescence.

626 The replicative lifespan reflects the number of buds generated by an individual mother cell.

627 Chronological lifespan: Time that non-dividing yeast cells in a stationary phase culture remain 628 viable.

629 Stationary phase: When nutrients are exhausted yeast cells enter a stationary phase that is

630 characterized by cell cycle arrest $\left(\mathrm{G}_{0}\right)$ and specific physiological, biochemical, and

631 morphological changes.

632 Respiratory capacity: Ability of yeast cells to grow on non-fermentable carbon sources, where

633 respiration (aerobic growth) is required.

634 [rho]: Deletions of the mitochondrial genome that render yeast cells respiratory-deficient (petite 635 phenotype).

636 Petite mutants: The yeast Saccharomyces cerevisiae can grow in the absence of mtDNA.

637 Yeast strains that contain wild-type mtDNA, called [rho $\left.{ }^{+}\right]$cells, can respire and grow on non-

638 fermentable carbon sources. Cells that contain deletions or mutations in mtDNA [rho] or have

639 completely lost their mtDNA [rho ${ }^{0}$ ] are called petite mutants. Petite mutants can grow by

640 fermentation in glucose media. Petite-negative cells lose viability in fermentable carbon sources.

641 Diauxic shift: S. cerevisiae switches metabolism from fermentation to respiration when growing

642 on glucose and in the presence of oxygen. During the first growth phase, when there is plenty of

643 glucose and oxygen available, yeast cells prefer glucose fermentation to aerobic respiration.

644 After glucose is depleted yeast cells undergo a metabolic (or diauxic) shift, where the 
645 fermentative product ethanol is oxidised. Diauxic shift is accompanied by stimulation of

646 mitochondrial function.

647 Nucleoids: Discrete protein-DNA complexes, organizing multiple mitochondrial DNA (mtDNA)

648 molecules.

649 Hereditary spastic paraplegia (HSP): Inherited neurological disorder characterized by

650 retrograde degeneration of cortical motor axons, progressive weakness (paraplegia), increased

651 muscle tone and stiffness (spasticity) of the legs. Loss of function of paraplegin (encoded by the 652 gene SPG7, a mitochondrial m-AAA-protease) causes HSP.

653 Leigh syndrome: Neurodegenerative disorder of infancy or childhood, generally due to

654 mutations in nuclear or mitochondrial genes involved in mitochondrial energy metabolism. 655 


\section{Figure legends}

656

657 Figure 1. Possible roles of the PHB complex in mitochondria. (a) Maintenance of mitochondrial membrane and cristae structure. The PHB complex is shown, interacting with mitochondrial inner and outer membrane proteins as part of a complex that might facilitate mitochondrial

660 fusion (e.g.: Stomatin, Stoml2/SLP-2) [42], Mitofusin (Mfn) [45], or others). The PHB complex

661 might also participate in the formation and/or maintenance of cristae junctions [5]. Additionally,

662 the PHB complex could have a role in keeping the two membranes of a crista in close proximity

663 (b) Biogenesis of OXPHOS complexes. The PHB complex may assist with protein folding and

664 assembly in cooperation with the m-AAA protease $[15,16]$. Association with mitochondrial

665 nucleoids [21-23] may ensure protection of highly hydrophobic mitochondrial-encoded OXPHOS

666 subunits until they are assembled with nuclear-encoded subunits into functional complexes. The

667 mitochondrial translocase of the outer membrane (TOM) and translocase of the inner

668 membrane (TIM) that mediate import of nuclear encoded mitochondrial proteins are also

669 depicted. The oxidative phosphorylation system (OXPHS) is schematically shown. The PHB

670 complex is represented as 12 heterodimers, each containing one PHB-1 and one PHB-2 (note

671 that given the predicted size of the complex, 12 to $16 \mathrm{PHB}-1 / \mathrm{PHB}-2$ heterodimers have been

672 proposed to assembled into the PHB complex) [17].

673

674 Figure 2. Involvement of the PHB complex in mitochondrial biology and cellular function. The

675 PHB complex has been proposed to play diverse roles within mitochondria (indicated by

676 arrows). Although the exact mechanism of action of prohibitins remains unknown, the

677 pronounced effects of prohibitin depletion in various organisms highlight the importance of this

678 evolutionarily conserved PHB protein complex.

679

680

681

682

683

684

685 
686

Figure 1

687
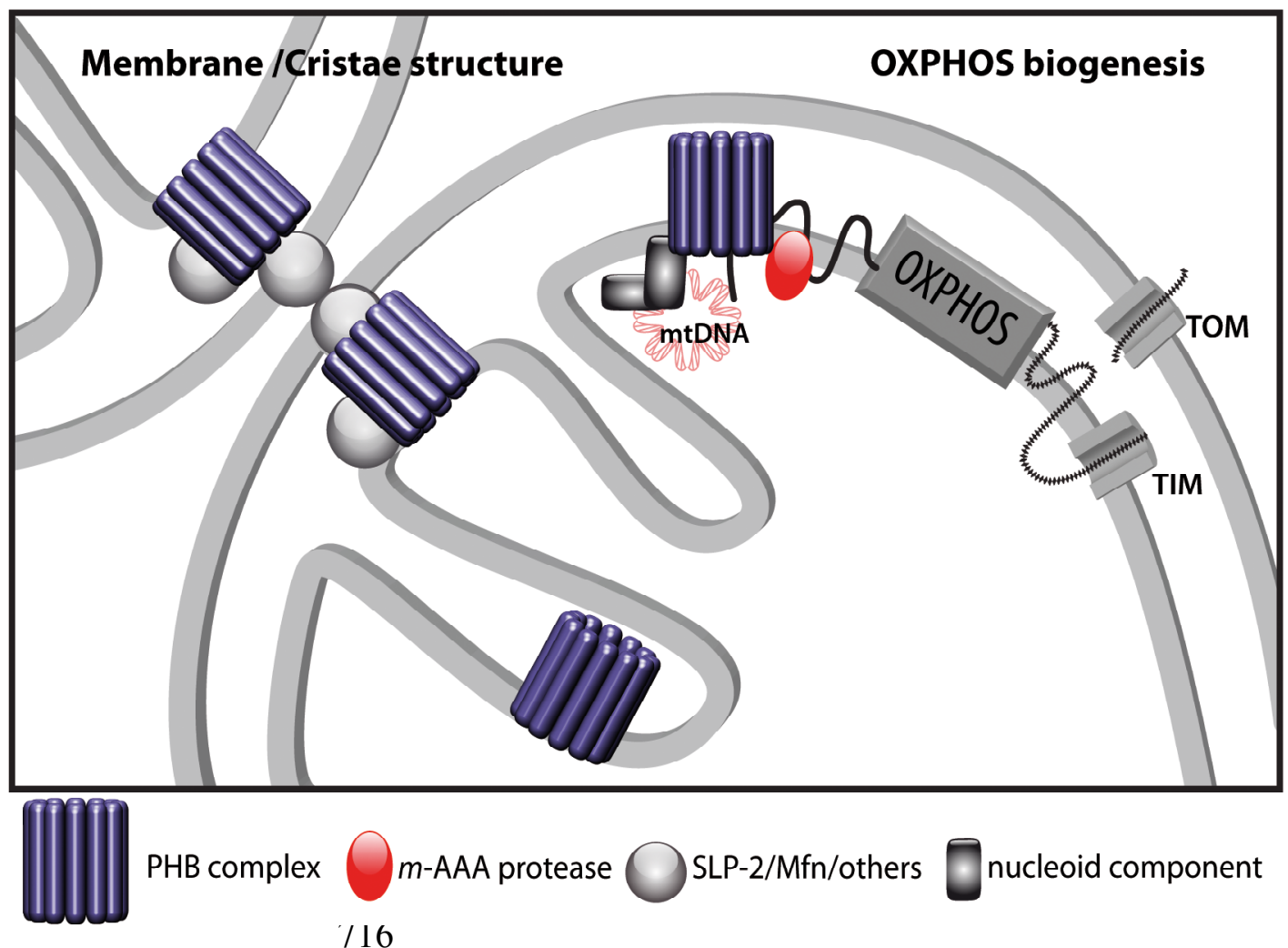

Figure 2

assembly/stabilisation of

726

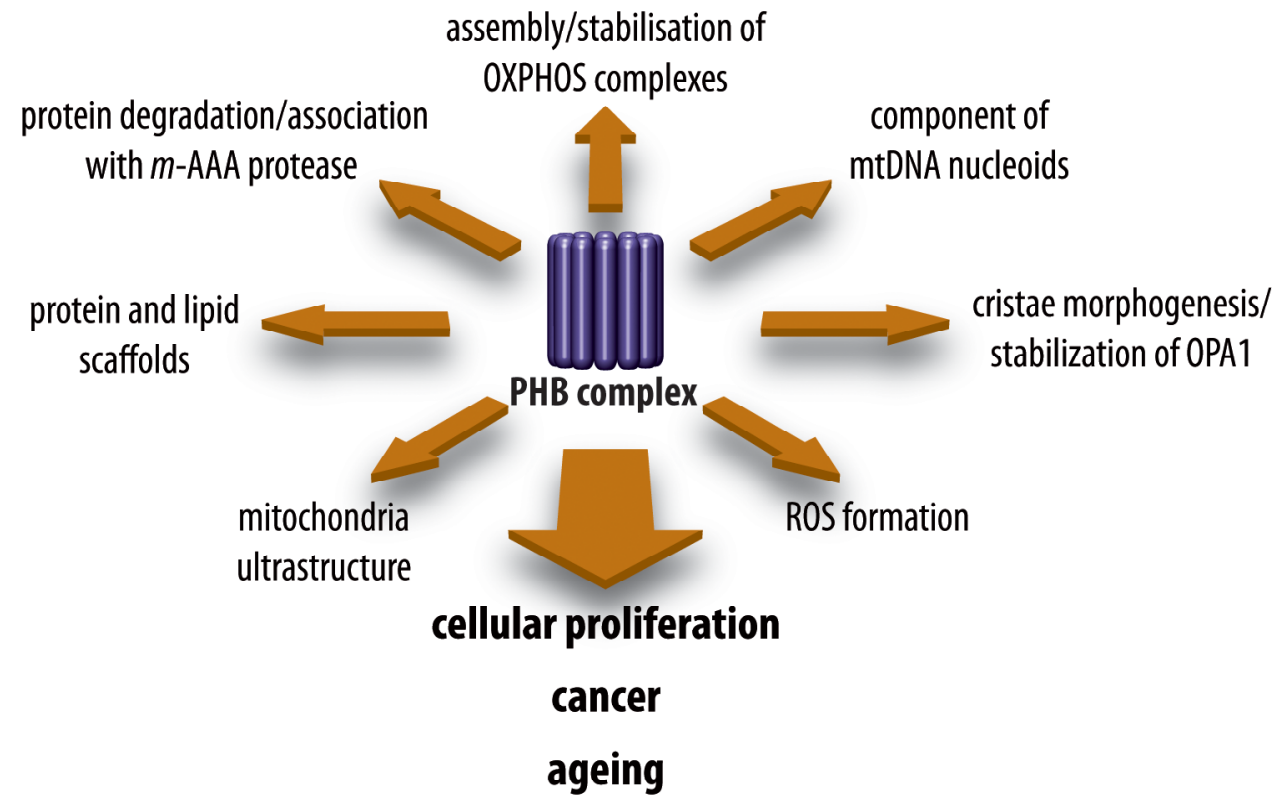

